\title{
A common cardiac sodium channel variant associated with sudden infant death in African Americans, SCN5A S1103Y
}

\author{
Leigh D. Plant, ${ }^{1}$ Peter N. Bowers, ${ }^{2}$ Qianyong Liu, ${ }^{1}$ Thomas Morgan, ${ }^{2}$ Tingting Zhang, ${ }^{1}$ \\ Matthew W. State, ${ }^{2}$ Weidong Chen, ${ }^{3}$ Rick A. Kittles, ${ }^{4}$ and Steve A.N. Goldstein ${ }^{1}$
}

'Department of Pediatrics and Institute for Molecular Pediatric Sciences, Pritzker School of Medicine, Biological Sciences Division, University of Chicago,
Chicago, Illinois, USA. ${ }^{2}$ Departments of Pediatrics, Genetics, and Child Study, Yale University School of Medicine, New Haven, Connecticut, USA.
${ }^{3}$ National Human Genome Center, Howard University, Washington, D.C., USA. ${ }^{4}$ Comprehensive Cancer Center, Ohio State University, Columbus, Ohio, USA.

\begin{abstract}
Thousands die each year from sudden infant death syndrome (SIDS). Neither the cause nor basis for varied prevalence in different populations is understood. While 2 cases have been associated with mutations in type $V \alpha$, cardiac voltage-gated sodium channels (SCN5A), the "Back to Sleep" campaign has decreased SIDS prevalence, consistent with a role for environmental influences in disease pathogenesis. Here we studied SCN5A in African Americans. Three of 133 SIDS cases were homozygous for the variant S1103Y. Among controls, 120 of 1,056 were carriers of the heterozygous genotype, which was previously associated with increased risk for arrhythmia in adults. This suggests that infants with 2 copies of S1103Y have a 24-fold increased risk for SIDS. Variant Y1103 channels were found to operate normally under baseline conditions in vitro. As risk factors for SIDS include apnea and respiratory acidosis, Y1103 and wild-type channels were subjected to lowered intracellular $\mathrm{pH}$. Only Y1103 channels gained abnormal function, demonstrating late reopenings suppressible by the drug mexiletine. The variant appeared to confer susceptibility to acidosis-induced arrhythmia, a gene-environment interaction. Overall, homozygous and rare heterozygous $S C N 5 A$ missense variants were found in approximately $5 \%$ of cases. If our findings are replicated, prospective genetic testing of SIDS cases and screening with counseling for at-risk families warrant consideration.
\end{abstract}

\section{Introduction}

Sudden infant death syndrome (SIDS) is the leading cause of infant mortality in the US among all racial and ethnic groups, representing nearly one-third of deaths between 1 month and 1 year of age (1). These losses continue despite dramatic improvement with the 1994 launch of the "Back to Sleep" campaign discouraging prone sleep for infants, a known risk factor for SIDS (2) that indicates a role for environmental stressors in the disorder. SIDS is defined as sudden and unanticipated death in an infant with no recognized lethal disorder after an investigation that includes autopsy, examination of the death scene, and review of the history (3). Suggesting a role for genetic predisposition, there is significant variation in SIDS among racial and ethnic groups; rates for African Americans and Native Americans are 3 times those for whites and 6 times higher than for Hispanics and Asians (1).

While multiple recognized risk factors suggest that SIDS is more than 1 entity and its pathogenesis multifactorial, the cardiac hypothesis $(4,5)$ has regained support due to identification in postmortem genetic samples from SIDS cases of mutations in the same ion channel genes that cause inherited and acquired long QT syndrome (LQTS) (6-11), 1 in a potassium channel (12) and 2 in a sodium channel (13). In addition to SIDS and LQTS, mutations in SCN5A cause Brugada syndrome, sudden unexplained nocturnal death syndrome (14), idiopathic ventricular fibrillation (15),

Nonstandard abbreviations used: LQTS, long QT syndrome; SCN5A, voltage-gated sodium channel, type $V \alpha$; SIDS, sudden infant death syndrome; $V_{1 / 2}$, voltage dependence of steady-state inactivation.

Conflict of interest: The authors have declared that no conflict of interest exists. Citation for this article: J. Clin. Invest. 116:430-435 (2006). doi:10.1172/JCI25618. congenital sick sinus syndrome (16), and progressive cardiac conduction defect (Lenegre-Lev disease) (17). Of note, many of these disorders are associated with bradycardia, the only consistent premorbid arrhythmia seen on cardiopulmonary monitors in SIDS cases immediately prior to death (18). Most SCN5A mutations causing arrhythmia produce channels that continue to operate late in the cardiac cycle when normal channels are silent; such late activity is a substrate for chaotic rhythms and sudden death (19-21). In African Americans, the common missense variant SCN5A S1103Y confers an 8-fold increased risk of arrhythmia without marked change in channel function in vitro (22). Genetic modulation of risk by exposure to extrinsic factors is seen in patients who carry potassium channel variants that are well tolerated until drug exposure produces arrhythmia $(10,11)$ and is consistent with a multi-hit pathogenesis for cardiac arrhythmia (23). To investigate the impact of changes in cardiac ion channel function on the risk of SIDS, samples were screened for genetic variations in SCN5A, and the attributes of variant channels were studied in the presence and absence of stressors and antiarrhythmic medications.

\section{Results}

Cohort and genetic analysis. The 224 autopsy-confirmed cases of SIDS in our case-control study reflect the expected distribution of SIDS cases in the US based on sex and ethnicity, with higher numbers of African Americans than whites (African American, 133; white, 86) and males than females (male, 106; female, 79). The control population for the African American SIDS cases consisted of 1,056 African American adults without known health problems. Twenty of the 133 African American SIDS cases had SCN5A variants that changed the channel protein (Supplemental Tables 1 and 2; 

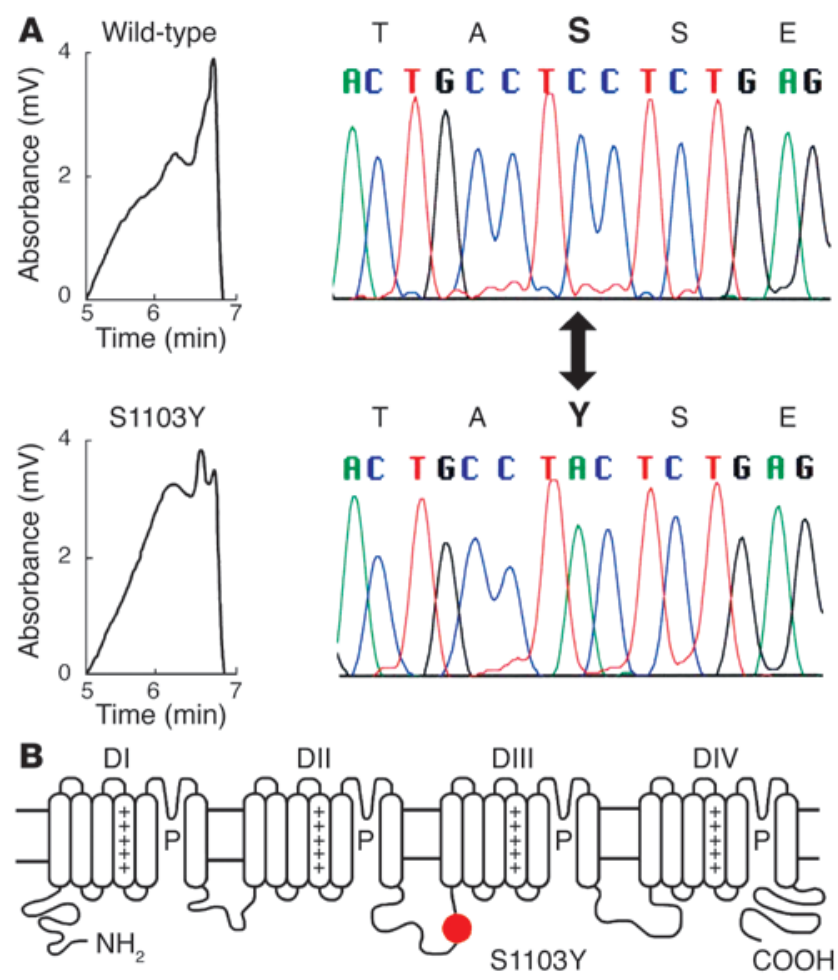

supplemental material available online with this article; doi:10.1172/JCI25618DS1). Thirteen cases were heterozygous for a common variant where a $\mathrm{C}$ replaces $\mathrm{A}$ in the second position of codon 1103 on 1 copy of SCN5A to yield a change from serine to tyrosine in the channel (Figure 1), indicating the genotype S1103/ Y1103 (SY). Three cases ( 2 female, 1 male) were homozygous for the variant genotype Y1103/Y1103 (YY), and so these infants carried only Y1103 channels. Four other cases had rare variants that changed a residue elsewhere in the channel on 1 SCN5A allele (Supplemental Figure 1). Of the 1,056 African American adult controls, the wild-type genotype S1103/S1103 (SS) was observed 935 times, SY was seen 120 times (similar to the previously reported frequency; refs. 22, 24), and 1 individual was homozygous (YY). This last individual could not be relocated to investigate for evidence of family history of SIDS or cardiac rhythm disturbances; he was 40 years of age and healthy at the time of sample collection.

Hardy-Weinberg equilibrium. Observed and expected genotypic frequencies, assuming Hardy-Weinberg equilibrium (HWE), for each genotype in African Americans were as follows. In SIDS cases $(n=133)$ : SS 117, 115 expected; SY 13, 17.6 expected; YY 3, 0.68 expected. In controls $(n=1,056)$ : SS 935, 938 expected; SY 120, 115 expected; YY 1, 3.52 expected. Case genotype frequencies were not in HWE $\left(\chi^{2}=9.21 ; P=0.002\right)$, whereas control frequencies did not significantly deviate from HWE $\left(\chi^{2}=2.04 ; P=0.15\right)$. In SIDS cases, there was a 4-fold excess of YY genotypes over the expected amount. In the controls, there was 1 YY individual, whereas 3 or 4 were expected. Comparing YY frequency in SIDS cases with controls, 3 of $133(2.3 \%)$ versus 1 of $1,056(0.1 \%)$, gives an odds ratio for SIDS of 24.4 (95\% confidence interval, 1.9 to 1,279), indicating an approximately 24 -fold increase in risk with the YY genotype. Overrepresentation of YY in SIDS cases was statistically significant, with a $P$ value of 0.005 by Fisher's exact test. To rule out misattribution in SIDS cases identified as African American, all SIDS

\section{Figure 1}

A polymorphism in SCN5A encodes a variant cardiac sodium channel. (A) Denaturing HPLC waveform and direct sequencing of wild-type and S1103Y variant. The chromatogram shows the additional peak resulting from heteroduplex assembly of S1103 and Y1103 amplicons. Forward sequence of homozygous S1103 and Y1103 SIDS cases shows the C3308A change that leads to the substitution of serine (S) by tyrosine (Y). (B) Topology of the cardiac sodium channel encoded by SCN5A shows the cytoplasmic location of the S1103Y missense change (red), the 4 homologous membrane domains (DI-DIV), the pore-forming $(P)$ loops, and the voltage-sensing segments $(+)$.

cases with SY and YY genotypes were evaluated and confirmed to bear the expected genetic ancestral proportions for Americans of African descent and were unrelated (Supplemental Table 1 and Supplemental Figure 2).

Y1103 channels inactivate abnormally at low pH. SCN5A encodes a 2016 residue $\alpha$ subunit with 4 similar domains (Figure 1B, DI-DIV) that fold in pseudosymmetric fashion to form a single, central ion permeation pathway lined by 4 pore loops. Position 1103 is in the intracellular linker between DII and DIII. To investigate channel operation, wild-type S1103 or Y1103 channel $\alpha$ subunits were expressed with the $\beta$ subunit of SCN5A, SCN1B, in mammalian tissue culture cells (HEK-293) (25). Channels were first studied using whole-cell patch-clamp mode so currents passed by many channels were assessed simultaneously. At pH 7.4, S1103 and Y1103 channels were found to function indistinguishably, showing the same midpoint for voltage-dependent activation (Figure 2A), midpoint for voltage-dependent steady-state inactivation (Figure 2B), and time course for recovery from inactivation (Figure 2C). Splawski and colleagues (22) observed subtle changes in gating of Y1103 channels under baseline conditions that were not apparent in our system. Hypothesizing that a more significant change in channel function might result from a challenge experienced in vivo, we assessed the effect of acidosis.

Because neither residue is charged, an amino acid change from $S$ to $\mathrm{Y}$ is not expected to produce $\mathrm{pH}$ sensitivity a priori. Nonetheless, lowering cytosolic $\mathrm{pH}$ (from 7.4 to 7.0 and 6.7) was found to induce progressive shifts in the voltage dependence of steady-state inactivation $\left(\mathrm{V}_{1 / 2}\right)$ for $\mathrm{Y} 1103$ channels (Figure 2, bottom row); thus, Y1103 showed $V_{1 / 2}$ to equal $-88 \pm 1,-84 \pm 2$, and $-77 \pm 2 \mathrm{mV}$ at $\mathrm{pH} 7.4,7.0$, and 6.7, respectively $(n=4-8)$. Wild-type S1103 channels did not show a similar change $\left(\mathrm{V}_{1 / 2}=-87 \pm 2,-88 \pm 1\right.$, and $-84 \pm 1 \mathrm{mV}$ at $\mathrm{pH}$ $7.4,7.0$, and 6.7, respectively; $n=7-21$ ) and so significantly differed with Y1103 at $\mathrm{pH} 6.7(P=0.005)$. In contrast, external acidification is expected to block peak current and shift steady-state inactivation (27), and these effects were observed for both channels to the same degree (Supplemental Table 3). Internal acidification was also found to speed recovery from inactivation of Y1103 but not wildtype SCN5A channels (Figure 2, bottom row). Changing the internal solution to $\mathrm{pH} 6.7$ led to a small decrease in recovery time for Y1103 channels that reached statistical significance $(P<0.05 ; n=11$ for each condition). Internal acidification also induced a small shift in the current/voltage relationship for Y1103 (Figure 2A, bottom inset) that did not achieve statistical significance.

These findings suggest Y1103 channels exposed to low internal $\mathrm{pH}$ might operate at times during the cardiac cycle when wildtype channels remain silent. To assess this hypothesis, whole-cell currents were recorded from groups of cells expressing S1103 channels with internal $\mathrm{pH} 7.4$ or 6.7 (Figure 3 , A and $\mathrm{B} ; n=10$ 
A

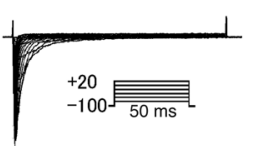

B
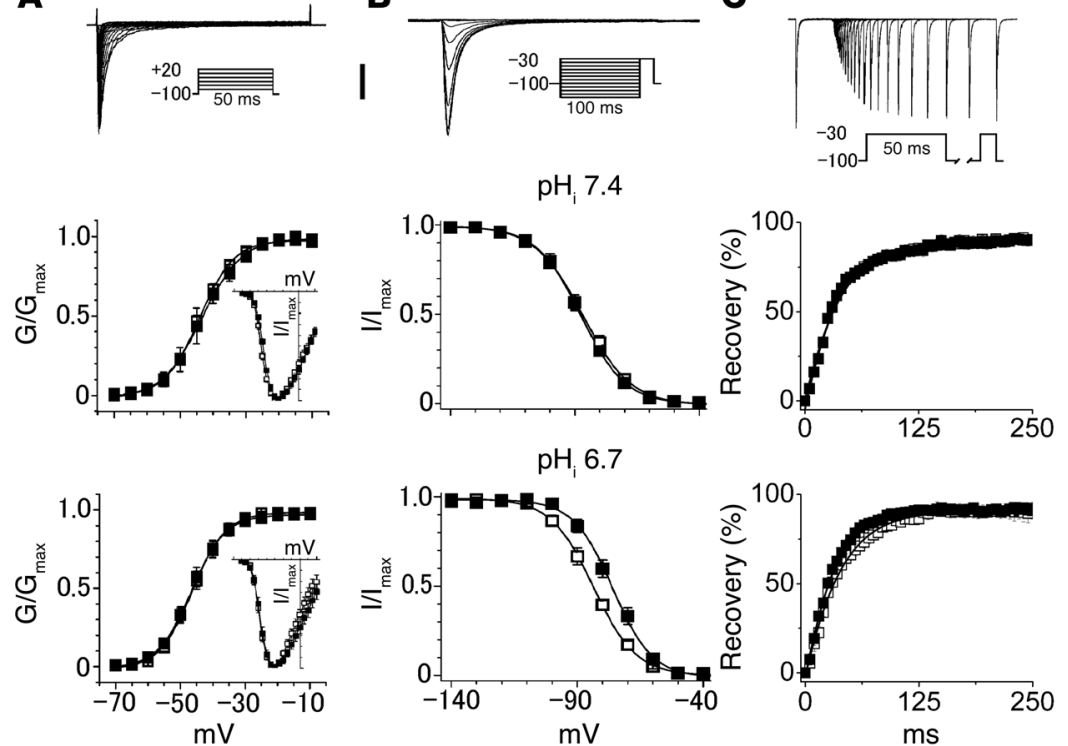

Figure 2

Y1103 channels show a change in inactivation gating at low $\mathrm{pH}$. Whole-cell currents in HEK-293 cells expressing SCN5A S1103 (open squares) or SCN5A Y1103 (filled squares). Top: Sample traces with $\mathrm{S} 1103$ channels. Arrows indicate points of measure. Scale bar: $1 \mathrm{nA}$. Filter and sampling frequencies were 5 and $20 \mathrm{kHz}$, respectively. (A) Normalized conductance/voltage $\left(G / G_{\max }\right)$ relationships for $\mathrm{S} 1103$ and $\mathrm{Y} 1103$ channels show no change with a shift of internal pH (pHi) from $\mathrm{pH} 7.4$ to $\mathrm{pH} 6.7(n=4-13)$. Curves fitted to a Boltzmann relationship $\left(y=1 /\left\{1+\exp \left[V-V_{1 / 2} / V s\right]\right\}\right.$, where $V s$ is slope factor). Inset: Current/voltage relationships. Values for $\mathrm{V}_{1 / 2}$ are reported in Supplemental Table 3. (B) Y1103 channels showed a shift in voltage-dependent steady-state inactivation at pHi 6.7, whereas $\mathrm{S} 1103$ channels did not. Normalized peak current values $\left(\mathrm{I} / \mathrm{I}_{\max }\right)$ are plotted against prepulse potential $(\mathrm{mV})$. Curves fitted as in $\mathbf{A}$. At pH 6.7, $\mathrm{V}_{1 / 2}$ for $\mathrm{S} 1103$ is $-84+1 \mathrm{mV}(n=7)$ and for $\mathrm{Y} 1103$ is $-77+2 \mathrm{mV}(n=8 ; P<0.05 ; 2$-population Student's $t$ test). Values are mean \pm SEM. (C) Time constants for recovery from inactivation were the same for $\mathrm{S} 1103$ and $\mathrm{Y} 1103$ channels at physiological $\mathrm{pH}$ but were speeded when $\mathrm{Y} 1103$, but not S1103, was exposed to pHi $6.7(n=11)$. Curves are exponential fits to the recovery time course, $\mathrm{I}(t) / I_{\max }=\mathrm{Af}[1-\exp (-t / \tau f)]+\mathrm{As}[1-\exp (-t / \tau s)]$, where $\mathrm{A}$ is the amplitude of fast (f) and slow (s) components and $t$ is time. Values for $\tau$ are reported in Supplemental Table 3.

cells for each condition). As expected, currents rapidly activated and inactivated to baseline; a double exponential fit revealed time constants of $1.8 \pm 0.04$ and $2.0 \pm 1.8 \mathrm{~ms}$ and $1.9 \pm 0.01$ and $1.8 \pm 0.8 \mathrm{~ms}$ at normal and low $\mathrm{pH}$, respectively. Conversely, $\mathrm{Y} 1103$ channels produced sustained whole-cell currents at low internal $\mathrm{pH}$ that did not return to baseline and showed altered inactivation kinetics (Figure 3, A and B). Late current was 5.23\% $\pm 0.85 \%$ of peak, and time constants were $2.5 \pm 0.01$ and $1.9 \pm 3.4 \mathrm{~ms}$ and $2.6 \pm 0.01$ and $79 \pm 0.6 \mathrm{~ms}$ at normal and low $\mathrm{pH}$, respectively ( $n=10$ cells, 50 sweeps/cell).

These changes in the voltage dependence of steady-state inactivation $(11 \mathrm{mV})$ and sustained current $(5 \%)$ with lowered $\mathrm{pH}$ were comparable to $2-6 \mathrm{mV}$ shifts and $1-3 \%$ sustained currents previously reported for $S C N 5 A$-dependent arrhythmia by others $(22,28,29)$. To confirm that late currents observed with low internal $\mathrm{pH}$ were carried by SCN5A rather than by other channel, transport, or leak pathways, single-channel function was studied.

Single Y1103 channels show abnormal late reopenings. Single wildtype cardiac sodium channels are closed at rest, open briefly with membrane depolarization, and then rapidly inactivate, staying silent despite maintained depolarization; the channels stay inactivated until the membrane repolarizes to resting potential, at which point they return to the closed state in preparation for the next heartbeat. A shift in the voltage dependence of inactivation with low $\mathrm{pH}$ suggests that single Y1103 channels may be active after depolarization, when wild-type channels are inactivated. As expected, single wild-type S1103 channels in inside-out patches opened just once on depolarization and then remained inactive at both pH 7.4 and pH 6.7 (Figure 4A); conversely, Y1103 channels operated normally at $\mathrm{pH} 7.4$ but showed abnormal reopenings during depolarization at $\mathrm{pH}$ 6.7. Repetitive cycles of stimulation of single channels averaged in cumulative fashion revealed that both the Y1103 change and low internal $\mathrm{pH}$ were required to induce sustained (late) current (Figure 4B).

Mexiletine suppresses pathological late reopenings. Bennett and colleagues demonstrated that SCN5A mutations associated with familial LQTS type 3 produce late channel reopenings without application of external stressors (8). These reopenings are blocked 5-10 times more effectively than initial openings (i.e., those required for normal cardiac function) by the channel blocker mexiletine, an antiarrhythmic used in adults and children (30). To assess drug suppression of Y1103 channels, a repetitive activation protocol approximating a heart rate of $150 \mathrm{bpm}$ was used (sodium channel block is sensitive to rate due to altered drug affinity in closed, open, and inactivated states). First, block of initial (peak) currents in whole-cell mode at pH 7.4 and 6.7 was studied. At pH 7.4, S1103 and Y1103 channels were inhibited similarly by propranolol and amiodarone (Supplemental Table 4), whereas Y1103 channels were somewhat more sensitive to mexiletine (Figure 5A, S1103 $\mathrm{K}_{1 / 2}=23.2 \pm 1.1 \mu \mathrm{M}$ [where $\mathrm{K}_{1 / 2}$ is the drug concentration yielding half maximal blockade], $\mathrm{Y} 1103 \mathrm{~K}_{1 / 2}=13.7 \pm 1.3 \mu \mathrm{M} ; n=8$ ). At $\mathrm{pH}$ 6.7, peak currents of Y1103 channels similarly remained sensitive to blockade $\left(\mathrm{K}_{1 / 2}=16.2 \pm 1.2 \mu \mathrm{M} ; n=8\right)$. To assess block of abnormal late reopenings, single Y1103 channels were studied at $\mathrm{pH}$ 6.7. As expected, application of $5 \mu \mathrm{M}$ mexiletine produced no significant suppression of initial activation (the null trace frequency was unaltered; Figure 5B), while nearly complete suppression of pathological late activity seen in the absence of drug (Figure 4B) was achieved (Figure 5B).

\section{Discussion}

The SCN5A Y1103 allele has been associated with arrhythmia and sudden death in 3 situations. African Americans with a single copy of the allele show an 8-fold increase in risk for arrhythmia as they reach middle age (22). Three siblings in a white kindred carrying a single allele have syncope, ventricular tachycardia, ventricular fibrillation, and sudden death (31). Here, homozygous African Americans infants carrying 2 Y1103 alleles showed a 24-fold increased risk for SIDS, with SIDS 


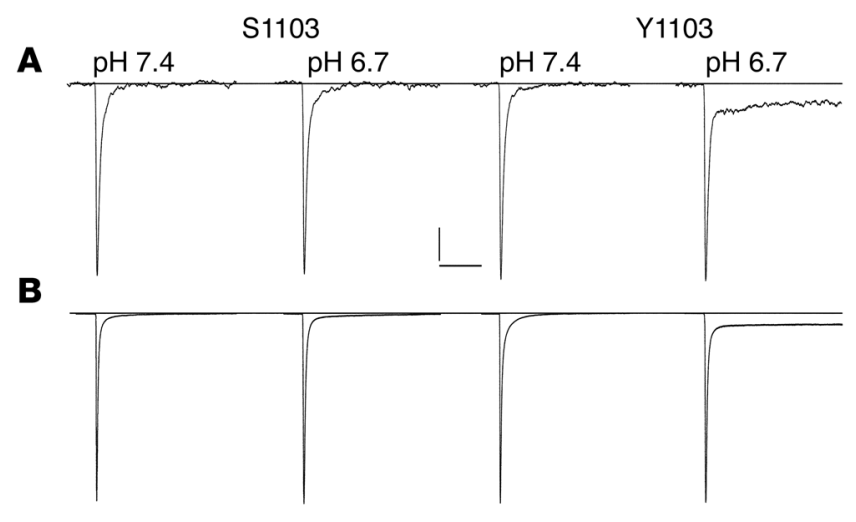

\section{Figure 3}

Cells expressing Y1103 channels show sustained current at a low pHi. Whole-cell currents were recorded in HEK-293 cells expressing $\mathrm{S} 1103$ or $\mathrm{Y} 1103$ channels as indicated with pipette and bath solutions (see Methods) with cells held at $-100 \mathrm{mV}$ and stepped to $-30 \mathrm{mV}$ for $100 \mathrm{~ms}$ at a rate of $0.5 \mathrm{~Hz}$. Scale bars represent $300 \mathrm{pA}$ and $20 \mathrm{~ms}$. (A) Representative currents recorded from cells at the indicated $\mathrm{pHi}$. (B) Ensemble averages of 500 sweeps recorded from 10 cells with the indicated channel and pHi. Late current was $5.23 \% \pm 0.85 \%$ of peak ( $n=10$ cells). Peak currents were not significantly different and were $-1311 \pm 143 \mathrm{pA}$ and $1252 \pm 139 \mathrm{pA}$, for $\mathrm{S} 1103$ at normal and low $\mathrm{pH}$, respectively, and $1267 \pm 173 \mathrm{pA}$ and $1195 \pm 245 \mathrm{pA}$ for Y1103 at normal and low pH, respectively ( $n=10$ cells per condition).

shown both tolerance of therapy and shortened QT $(38,39)$. These studies and our results suggest a strategy for prophylactic therapy, that is, use of state-dependent blockers that target late reopenings. The results do not indicate that mexiletine should be administered to YY infants to prevent SIDS at the present time. Should future work support our conclusion that YY infants are at significant risk, a study of prophylactic therapy is first required to assess benefits and risks. Moreover, it is important to note that the composition and environment of the channels we studied in cultured cells only approximate cardiac cells from SIDS patients; indeed, the identity of accessory with familio of Y1103 channels under baseline conditions was normal; this suggests that homozygous S1103Y infants will have a normal electrocardiogram in the absence of stressors and that this test would therefore not identify these genetically at-risk infants. Lastly, while the present report focuses on SCN5A S1103Y, the multifactorial nature of SIDS argues for mutation screening of other cardiac and noncardiac genes.

In this context, it is notable that many of the known risk factors for SIDS are stressors that can lead to prolonged apnea and respiratory acidosis - a common event in normal infants that may be exacerbated by diminished respiratory drive in situations including sleep, respiratory infection, multiple siblings (increasing infectious exposures), premature birth, and exposure to secondhand cigarette smoke. We speculate that increased risk for arrhythmia in aging African Americans with the Y1103 allele (22) may result from a similar mechanism, that is, low intracellular $\mathrm{pH}$ due to metabolic acidosis in poorly perfused cardiac muscle fed by an atherosclerotic coronary bed. Importantly, the internal $\mathrm{pH}$ levels of 7.0 and 6.7 used here to challenge Y1103 channels in vitro are relevant to cardiac physiology. While normal arterial $\mathrm{pH}$ is approximately 7.4, intracellular $\mathrm{pH}$ of cardiac myocytes is much lower. Cardiomyocytes in anesthetized, ventilated rats, rabbits, dogs, sheep, and ferrets have internal $\mathrm{pH}$ levels between 6.9 and 7.1 at baseline $(32,33)$. Internal pH decreases to 6.6-6.8 with respiratory acidosis and/or hypoxia $(34,35)$. Furthermore, acid loads dissipate slowly in myocardium, suggesting that basal microheterogeneity in intracellular $\mathrm{pH}$ will be pronounced under stress $(32,36)$.

It should be noted explicitly that suppression of abnormal function of Y1103 channels in vitro does not indicate in vivo effectiveness and that therapy with antiarrhythmic medication carries risk; conversely, mexiletine shortens the QT interval in animal models and adults with LQTS type 3 (37), and 2 infants with LQTS type 3 have been treated with mexiletine and

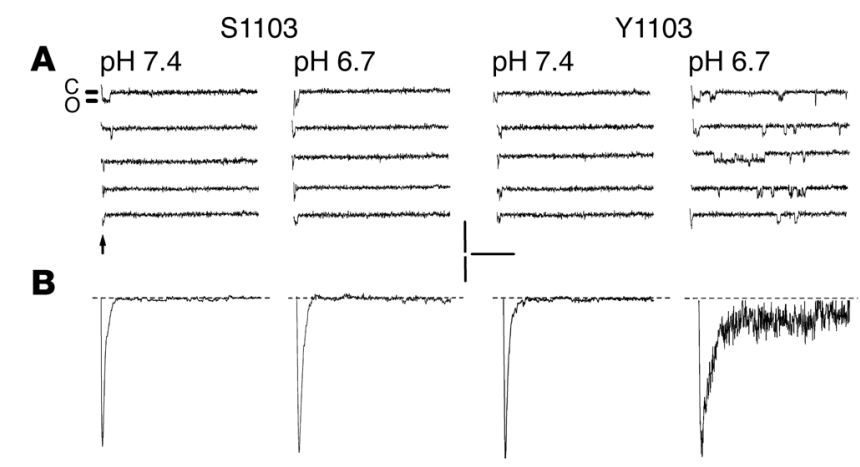

\section{Figure 4}

Single Y1103 channels show abnormal late reopenings. Single channels studied in inside-out, off-cell patches excised from HEK-293 cells. Currents were stimulated every 2.5 seconds by a $50-\mathrm{ms}$ depolarizing pulse to $-30 \mathrm{mV}$ from a holding potential of $-120 \mathrm{mV}$. Data were recorded at filter and sampling frequencies of 5 and $50 \mathrm{kHz}$, respectively. Pipettes were filled with bath solution described in Methods. Cells were perfused with the pipette solution described in Methods. For each cell, null sweeps (with no channel activity) were identified, averaged offline, and subtracted from data sweeps before analysis. For display purposes, data were refiltered offline using a $2-\mathrm{kHz}$ Bessel filter. (A) With depolarization, single $\mathrm{S} 1103$ channels opened, inactivated rapidly, and did not reopen. This behavior was unaltered when internal $\mathrm{pH}$ was lowered from 7.4 to 6.7. Null traces at $\mathrm{pH} 7.4$ and 6.7 were $50 \% \pm 2.7 \%(n=15$ patches, 1,367 sweeps) and $51 \% \pm 3.4 \%$ ( $n=12$ patches, 1,028 sweeps), respectively. In contrast, Y1103 channels behaved like S1103 channels at $\mathrm{pH} 7.4$ but showed late reopenings at $\mathrm{pH} 6.7$. Null traces were $51 \% \pm 4.5 \%$ at $\mathrm{pH} 7.4(n=8$ patches, 742 sweeps $)$ and $51.7 \pm 2.5 \%$ ( $n=10$ patches, 942 sweeps) when $\mathrm{pH}$ was reduced to 6.7. (B) Ensemble average traces ( $n=100-150$ sweeps) for the indicated channels and conditions. $Y 1103$ channels failed to remain in the inactivated state when exposed to internal pH 6.7. Scale bars: $0.5 \mathrm{pA} ; 10 \mathrm{~ms}$. 
A

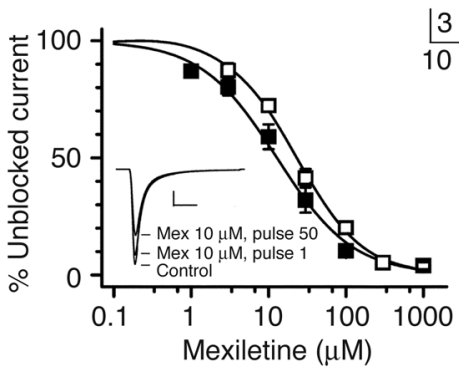

B

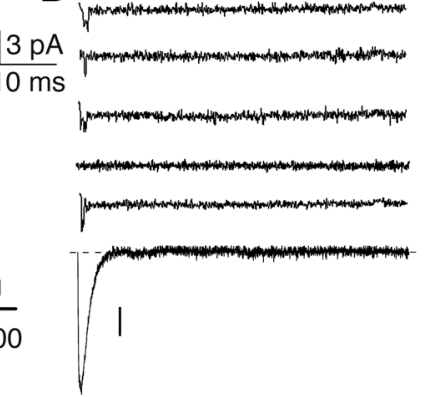

\section{Figure 5}

Abnormal reopenings are suppressed by mexiletine. Single channels were studied in inside-out, off-cell patches as in Figure 3. (A) Doseresponse curves for phasic block by mexiletine of peak macroscopic currents with $\mathrm{S} 1103$ (open squares) and Y1103 (filled squares) channels studied in whole-cell mode. Each point shows the average percent reduction in peak current by the dose indicated after a series of repetitive depolarizing stimuli ( $n=3-7$ cells). Cells were depolarized to $-30 \mathrm{mV}$ from a holding potential of $-100 \mathrm{mV}$ for $10 \mathrm{~ms}$ at $2.5 \mathrm{~Hz}$ to mimic a rate of $150 \mathrm{bpm}$. Inset: Representative $\mathrm{S} 1103$ current traces evoked by a pulse in drug-free solution (control) and the first (pulse 1) to show tonic block and fiftieth (pulse 50 ) to show phasic block by $10 \mu \mathrm{M}$ mexiletine (mex). Values for tonic and phasic block by mexiletine, propranolol, and amiodarone are reported in Supplemental Table 4. (B) Single Y1103 channels studied in inside-out off-cell patches showed that late reopenings of variant channels were fully suppressed by $5 \mu \mathrm{M}$ mexiletine. Null traces in the absence of drug (see Figure 3B) at pH 7.4 and 6.7 were $51 \% \pm 4.5 \%$ ( $n=8$ patches, 742 sweeps $)$ and $52 \pm 2.5 \%$ ( $n=10$ patches, 942 sweeps), respectively; null traces at $\mathrm{pH} 6.7$ with drug were $55 \% \pm 3.5 \%$ ( $n=6$ patches, 561 sweeps). The therapeutic blood level of mexiletine is $0.8-2.0 \mu \mathrm{g} / \mathrm{ml}(3.7-9.3 \mu \mathrm{M})$. Scale bars: $2 \mathrm{~ms}, 200 \mathrm{pA}$ (A); $0.3 \mathrm{pA}, 10 \mathrm{~ms}$ (B).

subunits in human sodium channels are still a subject requiring investigation (see ref. 40).

As with any association study, our findings will be need to be replicated in a separate cohort of African Americans. Even in relatively homogeneous genetic isolates, population stratification remains a potential explanation for false positive associations (41). However, the allele has been associated with cardiac arrhythmia in adults (22), and changes in function we observed here with low $\mathrm{pH}$ provide support for a plausible disease mechanism. The frequency of SIDS associated with changes in SCN5A channels in African Americans in our cohort was approximately 5\%. Thus, 7 of 133 SIDS cases were homozygous for YY or heterozygous for other residue changes in SCN5A channels. Changes at sites other than 1103 altered channel function at normal pH (S524Y, 2 cases; R689H and E1107K, 1 case each; Q. Liu and S.A.N. Goldstein, unpublished observations). Like individuals with inherited LQTS type 3, these infants had SCN5A gain in function in a single mutant allele. In contrast, African Americans homozygous for Y1103 were at increased risk for SIDS despite channels that appeared to be normal at baseline. The apparent etiology is arrhythmia due to a secondary challenge that is tolerated by others, perhaps respiratory acidosis due to hypoventilation. Genetic modulation of risk on exposure to common, extrinsic factors is recognized in patients who develop drug-induced arrhythmia due to mutations in potassium channels that are revealed upon drug exposure $(10,11)$ and is consistent with a multi-hit pathogenesis for cardiac arrhythmia (23).
This study leads us to consider genetic screening in African Americans for SCN5A S1103Y in at least 3 situations: infants with acute life-threatening events, siblings of SIDS victims, and couples that experience infertility or fetal demise (42). Given the apparent increased risk, a comprehensive study of postmortem molecular analysis of approximately 2,000 SIDS cases (the number reported each year in the US) is recommended. If replicated, prospective screening in a large cohort of African American infants should be considered. Finally, it is imperative to continue to support effective public health efforts to decrease known environmental risk factors (e.g., prone sleeping position). SIDS is argued by this work to result, like many common disorders, from a genetic predisposition that yields poor tolerance of common challenges to physiological homeostasis.

\section{Methods}

SIDS probands. Approval for human study protocols was obtained from the Human Investigations Committee of Yale University. Postmortem tissue was collected from 224 unrelated SIDS cases from 1985 to 1992 . Medical information was limited to sex, ethnicity, age at death, time of autopsy, and a brief history. Neither parental DNA nor family history were available. While cases were diagnosed as SIDS, it is not certain that all infants in our cohort died of SIDS because it is a diagnosis of exclusion with no definitive test. Apparently healthy adult African Americans served as controls; they were not screened for a family history of sudden death or SIDS.

Genomic DNA preparation, amplification, and mutational analysis. Genomic DNA was extracted from frozen heart, brain, or kidney blocks $\left(\sim 1 \mathrm{~cm}^{3}\right)$. Tissue was macerated on ice, and DNA was harvested by phenol/chloroform extraction, ethanol precipitation, and suspension in Tris-EDTA. The coding regions of SCN5A were amplified using primers and $\mathrm{PCR}$ conditions as shown in Supplemental Table 5. Denaturing HPLC heteroduplex analysis (WAVE; Transgenomic Corp.) was performed in the presence or absence of wild-type DNA, and variant samples were directly sequenced. For SCN5A S1103Y, all African American SIDS cases and controls were both screened by denaturing HPLC and directly sequenced. S1103Y was previously called S1102Y by Splawski et al. (22), whereas current mutation nomenclature for SCN5A now uses the long isoform NM_198056.1 (43).

Electrophysiology. Y1103 was introduced into SCN5A (NM_198056.1), the less abundant and longer isoform (43) carried in pcDNA1, using $P f u$ (Stratagene). Constructs were transiently cotransfected with GFPtagged SCN1B (the $\beta$ subunit of cardiac SCN5A channel, isoform b; NM_ 001037) in HEK-293 cells using lipofectamine (Invitrogen Corp.). After 24-60 hours, whole-cell recording was performed using a 200B amplifier and pCLAMP software (version 8.0; Axon Instruments). Voltage errors were minimized using $80 \%$ series resistance compensation. Whole-cell recording was performed using internal (pipette) and bath solutions, as previously described (22): internal, $60 \mathrm{mM} \mathrm{CsCl}, 80 \mathrm{mM} \mathrm{CsF}, 10 \mathrm{mM}$ EGTA, $1 \mathrm{mM} \mathrm{CaCl}_{2}, 1 \mathrm{mM} \mathrm{MgCl}_{2}, 5 \mathrm{mM} \mathrm{Na}_{2} \mathrm{ATP}$, and $10 \mathrm{mM}$ HEPES ( $\mathrm{pH}$ adjusted to indicate levels with $\mathrm{CsOH}$ ); bath, $130 \mathrm{mM} \mathrm{NaCl}, 5 \mathrm{mM}$ CsCl, $2 \mathrm{mM} \mathrm{CaCl}_{2}, 1.2 \mathrm{mM} \mathrm{MgCl}_{2}, 10 \mathrm{mM}$ HEPES, and $5 \mathrm{mM}$ glucose ( $\mathrm{pH} 7.4$ with $\mathrm{NaOH}$ ). Inside-out patch recording was performed using the same solutions, with the internal solution in the bath and the bath solution in the pipette. Mexiletine, propranolol, and amiodarone were from Sigma-Aldrich. Stock solutions were made in bath solution (mexiletine) or dimethyl sulfoxide (propranolol and amiodarone). Single channels were recorded in inside-out excised patches using borosilicate pipettes (Clark) coated with Sylgard (Dow Corning). All experiments were performed between $20-22^{\circ} \mathrm{C}$.

Statistics. In addition to estimation of odds ratios and exact $95 \%$ confidence limits, $P$ values were computed using Fisher's exact test, with a 
$P$ value less than 0.05 being considered statistically significant. HWE predictions were reported as expected versus observed for SIDS cases and controls using EpiInfo 2000 software (version 1.1.2; http://www.cdc. gov/epiinfo/). Electrophysiology data are reported as mean \pm SEM with ANOVA unless otherwise noted.

\section{Acknowledgments}

We thank the Brain and Tissue Banks for Developmental Disorders at the University of Maryland, our source of postmortem samples. We gratefully acknowledge the gift of SCN5A from R. Horn (Thomas Jefferson University, Philadelphia, Pennsylvania, USA) and SCN1B from A. George (Vanderbilt University, Nashville, Tennessee, USA). This work was supported by grants from the NIH and the Doris Duke Charitable Foundation to S.A.N.
Goldstein. We thank R. Goldstein for thoughtful discussions and Z. McCrossan for technical support.

Received for publication April 12, 2005, and accepted in revised form October 25, 2005.

Address correspondence to: Steve A.N. Goldstein, Department of Pediatrics and Institute for Molecular Pediatric Sciences, University of Chicago, Pritzker School of Medicine, 5721 South Maryland Avenue, Chicago, Illinois 60637, USA. Phone: (773) 702-6205; Fax: (773) 702-4523; E-mail: sangoldstein@uchicago.edu.

Peter N. Bowers, Leigh D. Plant, and Qianyong Liu contributed equally to this work.
1. Mathews, T.J., Menacker, F., and MacDorman, M.F. 2003. Infant mortality statistics from the 2001 period linked birth/infant death data set. Natl. Vital Stat. Rep. 52:1-28.

2. Hauck, F.R., et al. 2003. Sleep environment and the risk of sudden infant death syndrome in an urban population: the Chicago Infant Mortality Study. Pediatrics. 111:1207-1214.

3. Willinger, M., James, L.S., and Catz, C. 1991. Defining the sudden infant death syndrome (SIDS): deliberations of an expert panel convened by the National Institute of Child Health and Human Development. Pediatr. Pathol. 11:677-684.

4. Fearn, S.W. 1834. Two cases of sudden unexpected death of children during sleep. Lancet. 1:246-248.

5. Fraser, G.R., and Froggatt, P. 1966. Unexpected cot deaths [letter]. Lancet. 2:56.

6. Roden, D.M., et al. 1996. Multiple mechanisms in the long-QT syndrome - current knowledge, gaps, and future directions. Circulation. 94:1996-2012.

7. Goldstein, S.A.N., Keating, M., and Sanguinetti, M.C. 2004. Inherited cardiac arrhythmias. In Molecular basis of cardiovascular disease. K.R. Chien, editor. W.B. Saunders. Orlando, Florida, USA. 336-348.

8. Bennett, P.B., Yazawa, K., Makita, N., and George, A.L., Jr. 1995. Molecular mechanism for an inherited cardiac arrhythmia. Nature. 376:683-685.

9. Sanguinetti, M.C., Jiang, C., Curran, M.E., and Keating, M.T. 1995. A mechanistic link between an inherited and an acquired cardiac arrhythmia: HERG encodes the IKr potassium channel. Cell. 81:299-307.

10. Abbott, G.W., et al. 1999. MiRP1 forms IKr potassium channels with HERG and is associated with cardiac arrhythmia. Cell. 97:175-187.

11. Sesti, F., et al. 2000. A common polymorphism associated with antibiotic-induced cardiac arrhythmia. Proc. Natl. Acad. Sci. U. S. A. 97:10613-10618.

12. Schwartz, P.J., et al. 2001. Molecular diagnosis in a child with sudden infant death syndrome. Lancet. 358:1342-1343.

13. Ackerman, M.J., et al. 2001. Postmortem molecular analysis of SCN5A defects in sudden infant death syndrome. JAMA. 286:2264-2269.

14. Vatta, M., et al. 2002. Genetic and biophysical basis of sudden unexplained nocturnal death syndrome (SUNDS), a disease allelic to Brugada Syndrome. Hum. Mol. Genet. 11:337-345.

15. Chen, Q.Y., et al. 1998. Genetic basis and molecular mechanism for idiopathic - ventricular fibrillation. Nature. 392:293-296.

16. Benson, D.W., et al. 2003. Congenital sick sinus syndrome caused by recessive mutations in the car- diac sodium channel gene (SCN5A). J. Clin. Invest. 112:1019-1028. doi:10.1172/JCI200318062.

17. Schott, J.J., et al. 1999. Cardiac conduction defects associate with mutations in SCN5A. Nat. Genet. 23:20-21.

18. Meny, R.G., Carroll, J.L., Carbone, M.T., and Kelly, D.H. 1994. Cardiorespiratory recordings from infants dying suddenly and unexpectedly at home. Pediatrics. 93:44-49.

19. Spooner, P.M., et al. 2001. Sudden cardiac death, genes, and arrhythmogenesis: consideration of new population and mechanistic approaches from a National Heart, Lung, and Blood Institute workshop, Part II. Circulation. 103:2447-2452.

20. Gutstein, D.E., Danik, S.B., Sereysky, J.B., Morley, G.E., and Fishman, G.I. 2003. Subdiaphragmatic murine electrophysiological studies: sequential determination of ventricular refractoriness and arrhythmia induction. Am. J. Physiol. Heart Circ. Physiol. 285:H1091-H1096.

21. Kleber, A.G., and Rudy, Y. 2004. Basic mechanisms of cardiac impulse propagation and associated arrhythmias. Physiol. Rev. 84:431-488.

22. Splawski, I., et al. 2002. Variant of SCN5A sodium channel implicated in risk of cardiac arrhythmia. Science. 297:1333-1336.

23. Cheng, C.F., Kuo, H.C., and Chien, K.R. 2003. Genetic modifiers of cardiac arrhythmias. Trends Mol. Med. 9:59-66.

24. Ackerman, M.J., et al. 2004. Spectrum and prevalence of cardiac sodium channel variants among black, white, Asian, and Hispanic individuals: implications for arrhythmogenic susceptibility and Brugada/long QT syndrome genetic testing. Heart Rhythm. 1:600-607.

25. Xiao, Y.F., Wright, S.N., Wang, G.K., Morgan, J.P., and Leaf, A. 2000. Coexpression with beta(1)-subunit modifies the kinetics and fatty acid block of hH1(alpha) $\mathrm{Na}(+)$ channels. Am. J. Physiol. Heart Circ. Physiol. 279:H35-H46.

26. Daumas, P., and Andersen, O.S. 1993. Proton block of rat brain sodium channels. Evidence for two proton binding sites and multiple occupancy. J. Gen. Physiol. 101:27-43.

27. Zhang, J.F., and Siegelbaum, S.A. 1991. Effects of external protons on single cardiac sodium channels from guinea pig ventricular myocytes. J. Gen. Physiol. 98:1065-1083.

28. Wehrens, X.H.T., et al. 2003. A novel mutation in the cardiac $\mathrm{Na}+$ channel SCN5A associates with long-QT syndrome (LQT3): a role for the I-II linker in inactivation gating. Hum. Mutat. 21:552-560.

29. Keller, D.I., et al. 2003. A novel mutation in SCN5A,
delQKP 1507-1509, causing long QT syndrome. J. Mol. Cell. Cardiol. 35:1513-1521.

30. Wang, D.W., Yazawa, K., Makita, N., George, A.L., Jr., and Bennett, P.B. 1997. Pharmacological targeting of long QT mutant sodium channels. J. Clin. Invest. 99:1714-1720.

31. Chen, S., et al. 2002. SNP S1103Y in the cardiac sodium channel gene SCN5A is associated with cardiac arrhythmias and sudden death in a white family. J. Med. Genet. 39:913-915.

32. Vaughan-Jones, R.D., Peercy, B.E., Keener, J.P., and Spitzer, K.W. 2002. Intrinsic $\mathrm{H}(+)$ ion mobility in the rabbit ventricular myocyte. J. Physiol. 541:139-158.

33. Roos, A., and Boron, W.F. 1981. Intracellular pH. Physiol. Rev. 61:296-434.

34. Effros, R.M., et al. 1975. In vivo myocardial cell $\mathrm{pH}$ in the dog. Response to ischemia and infusion of alkali. J. Clin. Invest. 55:1100-1110.

35. Poole-Wilson, P.A., and Cameron, I.R. 1975. Intracellular $\mathrm{pH}$ and $\mathrm{K}+$ of cardiac and skeletal muscle in acidosis and alkalosis. Am. J. Physiol. 229:1305-1310.

36. Zaniboni, M., et al. 2003. Intracellular proton mobility and buffering power in cardiac ventricular myocytes from rat, rabbit, and guinea pig. Am.J. Physiol. Heart Circ. Physiol. 285:H1236-H1246.

37. Shimizu, W., and Antzelevitch, C. 1997. Sodium channel block with mexiletine is effective in reducing dispersion of repolarization and preventing torsade des pointes in LQT2 and LQT3 models of the long-QT syndrome. Circulation. 96:2038-2047.

38. Chang, C.C., et al. 2004. A novel SCN5A mutation manifests as a malignant form of long QT syndrome with perinatal onset of tachycardia/bradycardia. Cardiovasc. Res. 64:268-278.

39. Schulze-Bahr, E., et al. 2004. Long QT syndrome and life threatening arrhythmia in a newborn: molecular diagnosis and treatment response. Heart. 90:13-16.

40. Fahmi, A.I., et al. 2001. The sodium channel betasubunit SCN3b modulates the kinetics of SCN5a and is expressed heterogeneously in sheep heart. J. Physiol. 537:693-700.

41. Helgason, A., Yngvadottir, B., Hrafnkelsson, B., Gulcher, J., and Stefansson, K. 2005. An Icelandic example of the impact of population structure on association studies. Nat. Genet. 37:90-95.

42. Miller, T.E., et al. 2004. Recurrent third-trimester fetal loss and maternal mosaicism for long-QT syndrome. Circulation. 109:3029-3034.

43. Makielski, J.C., et al. 2003. A ubiquitous splice variant and a common polymorphism affect heterologous expression of recombinant human SCN5A heart sodium channels. Circ. Res. 93:821-828. 